\title{
Research into the Development of Service Model of Libraries in Colleges and Universities in Cloud Computing Environment
}

\author{
Ping Ni \\ The library of Hubei institute for nationalities, Enshi, Hubei 445000, China \\ nnpping@163.com
}

\section{Keywords: Cloud computing; Library; Service; Development}

\begin{abstract}
With the rapid development of information technology, all walks in the society begin to use the computer science and technology to improve their work efficiency, and in the cloud computing environment, service model of libraries in colleges and universities have also been changed. Not only services of libraries in colleges and universities have been updated and changed, but also the efficiency of utilizing resources in libraries in colleges and library users' degree of satisfaction have been improved. Based on this, this paper studies and analyzes the development of service model in libraries of colleges and universities in cloud computing environment.

With the emergence and development of computer network and cloud computing, traditional service model of libraries has been shocked and begin to transform into complex and digital libraries. Such evolution results from development of the network. At the current stage, cloud computing has brought major innovation to the development of libraries, so people begin to pay attention to cloud computing and new service model of libraries in cloud computing environment.
\end{abstract}

\section{Relevant Description of Cloud Computing}

Definition of Cloud Computing. Cloud computing integrates distributed computing, parallel computing and several carriers of grid computing, such as distributed management, parallel processing and grid computing, so it can be said that cloud computing is the manifestation of these concepts in the business field. Generally speaking, during the work process of cloud computing, users need to store relevant data in the Internet data center, instead of users' computers, and users' application programs are stored in the larger-scale Internet server instead of personal mobile terminal devices, such as computer and mobile phones, etc. Cloud computing providers are mainly responsible for maintaining data and managing the normal operation of these data so that users can have broader storage space and computing capacity under the background of cloud computing. Under this situation, users can use their data and materials more conveniently only by connecting the Internet during their work process in order to realize the low-configuration and high-performance services. In short, cloud computing refers to some service resources provided by the Internet service providers, including software resources and hardware resources so as to free users from the restriction of single computer and allow cloud computing to provide them with more services [1].

Principle of Cloud Computing. The storage level is mainly to store some of users' files and software in the database. The platform level has the identity recognition function because users need to use their usernames and passwords to $\log$ in. The second is collaborative operation, i.e. to allow services in cloud computing show a multi-threaded collaborative operation. The final is to send and receive information and operation of the application level should give certain response. Then, the application level is mainly to provide users with some concrete information, including several commonly seen applications, such as ERP, SCM and CRM, etc.

Types and Classification of Cloud Computing. In terms of the way of can provide service, cloud computing is mainly divided into public cloud, mixed cloud and private cloud. Public cloud is a cloud computing platform for the general public but they have to pay according to their demands. Contrary to public cloud, private cloud is not for the public system, but is the data center within certain organization or enterprise, so it is also called cloud computing platform mainly to integrate internal IT 
resources of enterprises. Mixed cloud has the advantages of both public cloud and private cloud, and it is also the general term of cloud computing serving for the cloud computing technology, and it can provide SAAS for cloud computing software and the main technique is the practical computing technology. Cloud computing is a revolution of the IT field with extensive influence on people's work and life as well as information technology, but as it is still at the beginning stage, there is still huge space for development of cloud computing. The following is research into various cloud computing services [3].

First of all, cloud service is officially called cloud model service, and there are two ways to define cloud service: one is to understand the basic characteristics of cloud service, that is to say, all the extensive services that can be provided by cloud can be called cloud service, such as the cloud computing service provided by Google, etc. Another one is the integration of traditional services, i.e. interpersonal services can be comprehensively integrated in the cloud computing model.

Cloud service provides a wide range of services and has its own service concept. That is to say, within a relatively large range, it is difficult to achieve local exchange and low recycling efficiency based on the overall situation. In this way, cloud computing analyzes specific problems and considers what service is suitable for what subject, i.e. to define the range and serving object so that it has advantage in exchange. For example, the process of urban development cannot go without the support of water and electricity, so such large-scale application like the water supply system and the power system are more suitable for the cloud service because they need to serve the entire city, and the characteristics of cloud service adapt to the requirements of the two systems, especially in resource-type services like water, natural gas and electricity, etc. From the perspective of human resource, this is also a resource-type service, so if human resource management is to be carried at a large scale, cloud service model can also be used, and if there are excessive human resources, enterprises need to pay high costs, so it is very suitable to use cloud service to solve this problem [4].

Secondly, the concept of cloud storage also develops on the basis of cloud computing and it is also an important part of cloud computing mainly referring to the application cluster, distributed files and network technology, etc. It can process a large amount of heterogeneous storage devices in the network with relevant software and then provide users with a system to visit the data storage business. In cloud computing, a core capacity is to provide users with a large amount of data for storage so as to assist users to comprehensively manage the system and utilize the hardware cluster and software cluster to consist the cloud storage system. The mainly task in the cloud system is to store and manage data.

\section{Theoretical Basis of Cloud Services in Libraries}

\section{Problems with Library Services.}

Libraries Lack Personalized Services. During the development, libraries have not used operational software that can provide personal services, and in the condition of modern information, in order to provide users with personalized and digital services, libraries need to understand users' demands so as to create a software system platform to serve users. This platform contains both hardware equipment and software equipment, and needs support of the Internet and manual maintenance more. This way can not only waste a lot of employees' time, but also consume a lot of materials, thus increasing costs of the library. In addition, during the process of establishing software platform, there are a large amount of complex functional modules and some useless software will be bonded when functional modules are bought, which will cost more resources and add costs for the enterprise again [5].

Problems with After-sales Services of Libraries' Integration System. At present, integration system is an indispensable part in library work because it can conduct library business and provide various library services. However, due to the development of information network, there are more and more software providers, thus after-sales service quality cannot be guaranteed because some software manufacturers only provide general telephone services and some manufacturers only provide online consulting services. What's worse, some small manufacturers do not provide after-sales services, so 
libraries need to spend both money and human resources to develop and maintain the libraries' integration system again. Meanwhile, managing staffs at libraries have varying capacity, so during the process of using integration system, the system's data may be lost or damaged, causing great trouble for library services.

Establishment and Sharing of Libraries. In order to reach the assessment standard, libraries will exert more efforts in purchasing books so that libraries, both large and small, can share resources, which is embodies not only in the operation mechanism, but also the game between each library. Then, there will be no alliance between libraries, thus the concept of sharing books will be delayed, and this repeated construction will cause great waste to both the country and the society.

\section{Cloud Services in Libraries.}

Definition of Cloud Services in Libraries. During the development of libraries, the so-called cloud service refers to starting from the service core and readers of libraries to provide cloud service to readers and constantly meet readers' various demands, including their demand for information, knowledge and literature, etc. In addition, with cloud service, libraries' internal and external resources can be constantly integrated to allow readers to experience more services, network community services and collaborative service. Meanwhile, libraries' cloud service can also use the cloud computing technology and put the specific service framework in the cloud terminal so that readers can freely use library resources and enjoy the convenience brought by cloud computing in libraries.

Principles of Libraries' Cloud Service. In order to establish their cloud computing platform, libraries need to comprehensively analyze principles of cloud services, but they should start from the principle of cloud computing in libraries, which are mainly manifested in the following aspects:

First of all, the orientation principle of cloud services in libraries. During the process of establishing cloud computing system in libraries, it is necessary for libraries to investigate users and readers, including readers' usage of libraries as well as their satisfaction towards libraries, etc. Only when libraries have had an all-round understanding of readers' behaviors can they establish the cloud computing services of libraries correctly. During the process of operating cloud services, libraries still need to pay timely attention to readers' changes and the difference between reader groups so as to correspondingly adjust and improve cloud service.

Secondly, the principle of system mainly regards libraries' cloud services as an integral part, which contains a lot of related factors, such as objects, contents and evaluation of cloud service, etc.

Thirdly, the sharing principle of cloud services in libraries involves sharing and applying library resources, so during the process of designing this system, libraries' service purpose should be implemented, the sharing and services should be combined to provide library service so as to form connection between books and literatures in the library with those in other libraries.

Finally, the expanding principle of cloud service in libraries mainly concern future development of libraries and lay a foundation for possible new services in cloud computing and cloud service, set useful hardware port and forecast new situation in setting cloud service, etc.

\section{Way to Construct Cloud Service Model in Libraries}

\section{Feasibility for Libraries to Use Cloud Service}

Feasibility and Safety for Libraries to Use Cloud Service. For libraries, the most significant advantage of cloud computing is it is safe and reliable. Cloud security is mainly in Internet system for the existence of a large number of mesh client software behavior monitoring, in order to obtain that may exist in the Internet security threats faced by Trojan or malicious software program, then these security information is sent to the server end of a comprehensive and systematic analysis and processing, and then the solution back to the Internet. This kind of security strategy for library cloud systems are protected by a certain extent, make it as far as against the invasion of harmful information to the greatest extent, reduce the possibility of user information exposed, this is cloud computing application in library service system the biggest advantage, can be enough to protect the information security. For example, some users of computer data loss, because maintenance result in data loss, but 
the use of cloud computing, the system will in the Internet, the clouds for the user's data and information is encrypted and reduce the possibility of the stolen data.

Feasibility and Compatibility of Cloud Services in Libraries. Compatibility of cloud computing mainly refers to that in libraries' cloud service, the early Internet service is combined with the current cloud computing service, and the server + client model is transferred to the service platform + client model. This framework of cloud computing becomes the Internet user data center, and frees users' behaviors from the previous computer desktop so as to allow users to enjoy various services at the Internet center. It can be said that the emergence of cloud computing is the progress and expansion of the Internet function. In this distributed model, cloud computing integrates hardware with software, and resolves the compatibility issue. The more important is the users' compatibility of the device. Cloud computing can be conducted on computers, mobile phones and IPAD so that users can break the restriction of time and space to access cloud resources in libraries.

\section{Overall Framework of Libraries' Cloud Service Model}

The application of cloud computing environment in libraries is actually a kind of technology revolution. In cloud service environment, libraries' internal resources and external resources are combined together so that traditional services are transformed into modern services. Basic framework of libraries' cloud service contains: cloud management and cloud intermediate. In cloud management, the host device is to establish the cloud platform and prepare cloud infrastructure as well as establish connection between data base and the main machine. In the framework of libraries' cloud service, there are work logs, user files and supporting platform as well as experience service, network community service, collaboration service and knowledge service in terms of user experience. Users include mobile reading, personal learning and personal digital library, etc.

\section{Introduction to Libraries' Cloud Service Model}

Cloud Fault of Libraries' Cloud Service Model. In order to realize the cloud service of libraries, it is necessary to set a device, cloud platform and server terminal at the terminal of library's cloud service and conduct multi-aspect management over corresponding library data. In cloud computing, there will be superior infrastructure cluster, which is the first choice for libraries to expand rapidly. The cloud terminal can process various problems in the libraries comprehensively to provide users with services. However, in order to obtain services provided by suppliers, libraries need to use the cloud intermediate, a carrier between libraries and suppliers mainly in charge coordinating relationship between the two. With the help of cloud intermediate, libraries can put their services onto the cloud terminal so as to provide all-round services for users.

Integration of Cloud Service Functions of Libraries. In the cloud computing environment, in order to provide users with diversified services, libraries need to comprehensively integrate their own functions and regard this as the basic target in the new situation so as to realize service innovation and technology application of libraries.

The first is user-experience service. Based on previous borrowing of books, this service also allows users to experience more feelings. For example, in terms of sharing of libraries' information resources and exchange, users can obtain information more freely to meet their demands. However, this is not the ultimate goal, and it is also necessary to allow exchange between libraries and users to provide help for reading so as to meet the demands of scientific research projects.

The second is the network community service. This service is mainly to allow cloud computing to provide a virtual service to make users have an all-round understanding of the establishment of libraries in the network community and give users a friendly library interface so as to optimize services.

\section{Conclusion}

To sum up, starting from the concept of cloud computing and cloud service, this paper elaborates on cloud services of libraries, hoping that through the support of modern science and technology as well as the application of cloud computing, great progress can be made towards libraries' functions. 


\section{References}

[1] Shangguang Wang,Zhipiao Liu,Qibo Sun et al.Towards an accurate evaluation of quality of cloud service in service-oriented cloud computing[J].Journal of Intelligent Manufacturing,2014,25(2):283-291.

[2] Min Min,Zhibo Zhang.On the influence of cloud fraction diurnal cycle and sub-grid cloud optical thickness variability on all-sky direct aerosol radiative forcing[J].Journal of Quantitative Spectroscopy \&amp; Radiative Transfer,2014,142:25-36.

[3] Tom Goren,Daniel Rosenfeld.Decomposing aerosol cloud radiative effects into cloud cover, liquid water path and Twomey components in marine stratocumulus[J].Atmospheric research,2014,138(Mar.):378-393.

[4] Wen, Y.,Zhu, X.,Rodrigues, J.J.P.C. et al.Cloud Mobile Media: Reflections and Outlook[J].IEEE transactions on multimedia,2014,16(4):885-902.

[5] Thamarai Selvi Somasundaram,Kannan Govindarajan.CLOUDRB: A framework for scheduling and managing High-Performance Computing (HPC) applications in science cloud[J].Future generations computer systems: FGCS,2014,34:47-65. 